\title{
Repercussão da perda de peso sobre parâmetros nutricionais e metabólicos de pacientes obesos graves após um ano de gastroplastia em Y-de-Roux
}

\author{
Nutritional and metabolic evaluation of patients after one year of gastric \\ bypass surgery
}

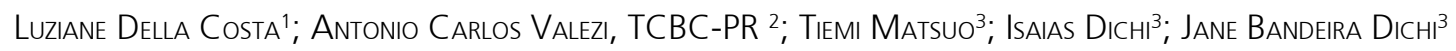

R E S U M O

\begin{abstract}
Objetivo: Avaliar a evolução metabólico-nutricional e a atividade inflamatória em pacientes com obesidade grave submetidos à cirurgia bariátrica. Métodos: Realizou-se um estudo prospectivo em 56 pacientes (50 mulheres e seis homens), apresentando média de idade de 40 +/- 9,9 anos, submetidos à RYGB. Avaliação metabólica e nutricional e da atividade inflamatória foram verificadas antes, seis e 12 meses após o procedimento cirúrgico. Resultados: Verificou-se redução significativa nos valores iniciais, em relação à perda de peso de $138 \pm 28,8$ to $90 \pm 19,5 \mathrm{~kg}(\mathrm{p}<0,0001)$, glicemia de $116 \pm 47,3$ to $84 \pm 9,8 \mathrm{mg} / \mathrm{dL}(\mathrm{p}<0,0001)$, níveis de triacilglicerol de $137 \pm 61,4$ to $84 \pm 38,6 \mathrm{mg} / \mathrm{dL}(p<0,0001)$, colesterol total de $189 \pm 41,6$ to $166 \pm 36,4 \mathrm{mg} / \mathrm{dL}(p<0,0001)$ e LDLcolesterol de $119 \pm 36,1$ para $104 \pm 30,7 \mathrm{mg} / \mathrm{dL}$ ( $p<0,0005$ ). Os níveis de proteína C-reativa reduziram de 11,33 $\pm 10,82$ para 3,62 $\pm 4,49 \mathrm{mg} / \mathrm{dL}(\mathrm{p}<0,0001)$. Embora os níveis de ferro tenham permanecido dentro do limite de normalidade, após um ano, observouse diminuição significativa na hemoglobina de $13 \pm 1,3$ para $12 \pm 1,4 \mathrm{~g} / \mathrm{dL}(\mathrm{p}<0,01)$, e redução nos níveis de ferritina, particularmente nas mulheres, que apresentou queda de 101,2 $\pm 123,3$ para 85,0 $\pm 101,9 \mathrm{~g} / \mathrm{dL}(\mathrm{p}<0,03)$. Conclusão: A melhora verificada no estado metabólico e inflamatório concomitantemente após tratamento cirúrgico pode reduzir substancialmente as co-morbidades associadas com o risco cardiovascular aumentado.
\end{abstract}

Descritores: Obesidade. Gastroplastia. Perda de peso. Dislipidemia. Proteína C-reativa.

\section{INTRODUÇÃO}

A obesidade é considerada uma doença crônica, de etiologia multifatorial, cujo excesso de adiposidade está relacionado à pré-disposição genética e, principalmente aos fatores ambientais. Monteiro et al. ${ }^{1}$ verificaram aumento na prevalência de sobrepeso e obesidade na população adulta brasileira.

Sabe-se que o excesso de gordura corpórea está associado com risco elevado de doença cardíaca isquêmica, acidente vascular cerebral, hipertensão arterial, diabetes melito tipo 2, dislipidemia, osteoartrite e apnéia obstrutiva do sono, contribuindo dessa forma para aumentar a morbidade e mortalidade nestes pacientes ${ }^{2}$.

Opções clássicas, não cirúrgicas para conquistar perda de peso, em pacientes obesos, incluem restrição dietética, atividade física, modificações comportamentais, medicamentos e suporte psicológico, cujos resultados, frequentemente são limitados e obtidos em curtos períodos de tempo ${ }^{3}$.

Atualmente, terapia cirúrgica para tratar obesidade grave é considerada a melhor maneira para reduzir o excesso de peso corpóreo concomitantemente com melhora na qualidade de vida em vários estudos no longo prazo, pois contribui para diminuir as co-morbidades relacionadas à obesidade, principalmente em pacientes selecionados ${ }^{4}$. Além disso, pacientes obesos submetidos ao tratamento cirúrgico apresentam redução na mortalidade total e menor risco de desenvolver doenças cardiovasculares, neoplasias, alterações endócrinas, anormalidades psiquiátricas e mentais quando comparados aos obesos que não tiveram oportunidade de serem submetidos à correção cirúrgica de obesidade 5 .

Há várias opções cirúrgicas disponíveis para tratamento de obesidade, mas a gastroplastia vertical em $Y$ de Roux (GVYR) é considerada como padrão ouro para que o paciente consiga uma perda de peso sustentável [6], haja vista que associa restrição gástrica com algum grau de má absorção. Buchwald et al. ${ }^{7}$, em um estudo de meta-análise, concluíram que este procedimento cirúrgico suprime ou melhora a intolerância gástrica e/ ou diabetes, dislipidemia, hipertensão, e apnéia do sono. Além disso, pessoas com IMC elevado podem apresentar também nível no sangue de proteína C-reativa (PCR) alto e, dessa forma, a perda de 
peso acarretaria não só redução de PCR como também de interleucina-1 concomitantemente à diminuição na resistência periférica à insulina, diminuindo o risco de doença cardiovascular $^{8}$. Sabe-se que, embora ocorra melhora clínica após tratamento cirúrgico, o paciente necessitará de acompanhamento nutricional regular com o objetivo de promover uma reeducação alimentar para a aquisição de hábito dietético saudável, prevenindo deficiências de vitaminas e micronutrientes ${ }^{9}$.

O objetivo do presente estudo é avaliar, em pacientes com obesidade grave submetidos à gastroplastia vertical em Y de Roux, os efeitos da perda de peso sobre a glicemia, o perfil de lipídios, o metabolismo do ferro e a atividade inflamatória.

\section{MÉTODOS}

O objetivo do presente estudo é avaliar, em pacientes com obesidade grave submetidas à gastroplastia vertical em $Y$ de Roux, os efeitos da perda de peso sobre a glicemia, o perfil de lipídios, o metabolismo do ferro e a atividade inflamatória.

Todos os pacientes assinaram termo de consentimento e o protocolo de estudo foi totalmente aprovado pelo Comitê de Ética da Universidade Estadual de Londrina.

Realizou-se estudo prospectivo, clínico-nutricional, envolvendo 56 pacientes, 50 mulheres (89\%) e seis homens (11\%), média de idade de 40,2 $\pm 9,9$ anos, submetidos à gastroplastia vertical em $Y$-de-Roux, no Serviço de Cirurgia do Aparelho Digestivo do Hospital Universitário de Londrina, Paraná.

Todos os pacientes foram avaliados, mediante parâmetros clínicos, laboratoriais e nutricionais, antes do procedimento cirúrgico e após seis e 12 meses. Avaliação nutricional foi realizada mediante questionário de freqüência alimentar e índice de massa corpórea $\left(\mathrm{IMC}\left(\mathrm{kg} / \mathrm{m}^{2}\right)=\right.$ peso/estatura ${ }^{2}$ ), e os pacientes classificados de acordo com recomendação da Organização Mundial de Saúde ${ }^{10}$.

Após jejum de 12 horas, os pacientes foram submetidos aos seguintes exames laboratoriais sanguíneos: hemoglobina (automated counter - Pentra 120 Retic ABX), ferro, transferrina e ferritina (Método Ferrozine com analizador químico automático - Dimension AR, DadeBehring), glicose, colesterol total (colesterol oxidase), HDLcolesterol (precipitação seletiva) e triacilglicerol (lipase/ glicerol dehidrogenase - auto-analisador bioquímico Dade $A R)$; LDL-colesterol (calculado pela equação de Friedwald); albumina (Método do verde de bromcresol) e proteína Creativa (nefelometria).

Todos os dados estão apresentados como média \pm desvio padrão. As variáveis medidas nos diferentes momentos do estudo foram testadas pela ANOVA, sendo o valor de $P<0,05$ considerado como estatisticamente significante.

\section{RESULTADOS}

Após a gastroplastia em $Y$ de Roux, foram observadas várias mudanças na ingestão alimentar habitual dos pacientes, haja vista que muitos relatavam diminuição na ingestão de arroz, feijão, doces, pães e ovos, e ingestão aumentada de biscoitos, frutas e sucos de fruta. Além disso, houve relatos de intolerância à carne, especialmente carne vermelha, passando a preferir frango, enquanto a ingestão de leite permaneceu inalterada (dados não mostrados).

Com relação aos valores do peso inicial (138 \pm $28,8 \mathrm{~kg})$, verificou-se significante $(p<0,0001)$ perda de peso após seis $(102 \pm 22,4 \mathrm{~kg})$ e 12 meses $(90 \pm 19,5 \mathrm{~kg})$, sendo que o mesmo comportamento foi observado em relação ao IMC que diminuiu, significantemente $(p<0,0001)$, passando de $52 \pm 8,6 \mathrm{~kg} / \mathrm{m}^{2}$, antes da cirurgia para $39 \pm 7,3$ após seis e $34 \pm 6,6 \mathrm{~kg} / \mathrm{m}^{2}$ após 12 meses de cirurgia (Tabela 1).

Tabela 1 - Parâmetros do estado nutricional e os valores de proteína C-reativa (Média土DP) antes, seis e doze meses após "bypass" gástrico*.

\begin{tabular}{|c|c|c|c|c|c|c|}
\hline Parâmetros & Pré o & ratório & Pós operatc & rio 6 meses & Pós operató & rio 12 meses \\
\hline Peso $(\mathrm{kg})$ & $138 \pm$ & 28,8 & $102 \pm$ & $22,4^{a}$ & $90 \pm$ & $19,5^{b}$ \\
\hline $\mathrm{IMC}\left(\mathrm{kg} / \mathrm{m}^{2}\right)$ & $52 \pm$ & 8,6 & $39 \pm$ & $7,3^{\text {a }}$ & $34 \pm$ & $6,6^{b}$ \\
\hline Glicose $(\mathrm{mg} / \mathrm{dL})$ & $116 \pm$ & 47,3 & $85 \pm$ & $12,0^{a}$ & $84 \pm$ & $9,8^{b}$ \\
\hline Colesterol Total (mg/dL) & $189 \pm$ & 41,6 & $169 \pm$ & $37,9^{a}$ & $166 \pm$ & $36,4^{b}$ \\
\hline Colesterol HDL (mg/dL) & $41 \pm$ & 10,3 & $39 \pm$ & 10,0 & $43 \pm$ & 10,4 \\
\hline Colesterol LDL (mg/dL) & $119 \pm$ & 36,1 & $110 \pm$ & $28,9^{c}$ & $104 \pm$ & $30,7^{d}$ \\
\hline Triacilglicerol (mg/dL) & $137 \pm$ & 61,4 & $99 \pm$ & $43,1^{\text {a }}$ & $84 \pm$ & $38,6^{b}$ \\
\hline Albumina $(\mathrm{g} / \mathrm{dL})$ & 3,63 & 0,33 & 3,73 & $\pm 0,37$ & 3,75 & $\pm 0,30$ e \\
\hline Proteína C- reativa $(\mathrm{mg} / \mathrm{dL})$ & 11,33 & $: 10,82$ & 5,96 & $\pm 5,95^{c}$ & 3,62 & $\pm 4,49^{b}$ \\
\hline
\end{tabular}

* Número de pacientes = 56; IMC = índice de massa corporal

a Diferença significativa nos valores pré-operatórios vs 6 mo $(P<0,0001)$

${ }^{b}$ Diferença significativa nos valores pré-operatórios vs 12 mo $(P<0,0001)$

c Diferença significativa nos valores pré-operatórios vs 6 mo $(P=0,03)$

${ }^{d}$ Diferença significativa nos valores pré-operatórios vs 12 mo $(P<0,0005)$

e Diferença significativa nos valores pré-operatórios vs 12 mo $(P<0,01)$ 
Em relação aos níveis de glicemia, verificou-se redução com significância estatística $(\mathrm{p}<0,0001)$ nos valores basais que eram de $116 \pm 47,3 \mathrm{mg} / \mathrm{dL}$ para $85 \pm 12,0 \mathrm{e}$ $84 \pm 9,8 \mathrm{mg} / \mathrm{dL}$ após seis e 12 meses, respectivamente. Este mesmo comportamento foi observado em relação ao colesterol total, cujos valores diminuíram, de modo significante $(p<0,0001)$ a partir do basal que era de $189 \pm 41,6 \mathrm{mg} / \mathrm{dL}$ para $169 \pm 37,9$ e $166 \pm 36,4 \mathrm{mg} / \mathrm{dL}$ após seis e 12 meses, respectivamente, após a gastroplastia. Por sua vez, o nível de LDL-colesterol mostrou significante redução de $119 \pm 36,1 \mathrm{mg} / \mathrm{dL}$ para $110 \pm 28,9(p=0,03)$ e $104 \pm 30,7 \mathrm{mg} / \mathrm{dL}(\mathrm{p}=0,0005)$ após seis e 12 meses, respectivamente, em relação ao nível basal. Além disso, os níveis de triacilglicerol também apresentaram queda significante $(p<0,0001)$ de $137 \pm 61,4 \mathrm{mg} / \mathrm{dL}$ inicialmente para $99 \pm 43,1$ e $84 \pm 38,6 \mathrm{mg} / \mathrm{dL}$, após seis e 12 meses, respectivamente. Entretanto, em relação ao HDL-colesterol, não houve diferença estatística entre os valores iniciais e após seis e 12 meses (Tabela 1).

Os valores basais de albumina plasmática $(3,63 \pm 0,33 \mathrm{~g} / \mathrm{dL})$ aumentaram após seis meses $(p=0,09)$, porém, aumento com significância estatística $(p=0,01)$ ocorreu, somente após 12 meses de estudo $(3,75 \pm 0,3 \mathrm{~g} / \mathrm{dL})$. Entretanto, os níveis de proteína C-reativa mostraram redução significante após seis $(p=0,03)$ e 12 meses $(p<0,0001)$, passando de $11,33 \pm 10,82 \mathrm{mg} / \mathrm{dL}$ no momento inicial para $5,96 \pm 5,95$ e 3,62 $\pm 4,49$, respectivamente (Tabela 1).
Os resultados de hemoglobina, índices hematimétricos e do metabolismo do ferro encontram-se na tabela 2. Quando comparados aos valores basais, verificou-se redução significante $(p=0,01)$ na hemoglobina de $13 \pm 1,27 \mathrm{~g} / \mathrm{dL}$ para $12 \pm 1,36$ e $12 \pm 1,39 \mathrm{~g} / \mathrm{dL}$, respectivamente, aos seis e 12 meses após a cirurgia, e na contagem de plaquetas cujos valores eram de $300340 \pm 77967 \mathrm{n} \%$ $\mathrm{mm}^{3}$ e passaram para $285170 \pm 69863$ e $266979 \pm 60354$ $\mathrm{n} / \mathrm{mm}^{3}$, respectivamente, aos seis $(p=0,05)$ e 12 meses $(p=0,001)$ após a operação.

Após um ano da realização da gastroplastia em $Y$ de Roux, foi verificado também aumento significante ( $p$ $=0,05)$ no nível sanguíneo de transferrina em relação aos valores basais, isto é de $261 \pm 85,2$ para $285 \pm 85,1 \mu \mathrm{g} / \mathrm{dL}$.

O nível sérico de ferro, após um ano de cirurgia não mostrou qualquer alteração significante, entretanto os níveis de ferritina sérica diminuíram, passando de $125 \pm$ $196,9$ para $87 \pm 118,4 \mu \mathrm{g} / \mathrm{L}(\mathrm{p}=0,08)$ (Tabela 2$)$. Em relação à avaliação dos níveis séricos de ferritina, específicamente entre as mulheres que participaram deste protocolo, verificou-se redução, estatisticamente significante $(p=0,03)$, de 101,2 $\pm 123,3$ para $89,7 \pm 100,7$ após seis meses e para 85,0 $\pm 101,9$ após um ano (dados não mostrados).

A tabela 3 mostra redução progressiva na frequência de vários fatores de risco de doença cardiovascular, tais como IMC, glicemia, HDL-colesterol, e

Tabela 2 - Parâmetros dos valores sanguineos antes e após a operação .

\begin{tabular}{|c|c|c|c|c|}
\hline Parâmetros & Pré op & operatório & Pós operatório 6 meses & Pós operatório 12 meses \\
\hline Hemoglobina ( $\mathrm{g} / \mathrm{dL})$ & $13 \pm$ & 1,3 & $12 \pm 1,4^{a}$ & $12 \pm 1,4^{b}$ \\
\hline Hematócrito (\%) & $38 \pm$ & 4,7 & $37 \pm 4,0$ & $37 \pm 4,2$ \\
\hline $\operatorname{VCM}(f \mathrm{~L})$ & $84 \pm$ & 6,8 & $83.41 \pm 5,7$ & $84 \pm 5,4$ \\
\hline HCM (pg) & $27 \pm$ & 2,4 & $27 \pm 2,3$ & $28 \pm 2,3$ \\
\hline $\operatorname{CHCM}(\%)$ & $33 \pm$ & 0,8 & $33 \pm 0,9$ & $33 \pm 0,7$ \\
\hline Plaquetas (n/m³ $\square 10^{4}$ ) & $30,0 \pm$ & 7,8 & $28,5 \pm 7,0^{d}$ & $26,7 \pm 6,0^{c}$ \\
\hline Ferro $(\mu \mathrm{g} / \mathrm{L})$ & $67 \pm$ & $\pm \quad 31,6$ & $65 \pm 28,0$ & $67 \pm 28,7$ \\
\hline Ferritina $((\mu \mathrm{g} / \mathrm{L})$ & $125 \pm$ & $\pm 196,9$ & $96 \pm 116,7$ & $87 \pm 118,4$ e \\
\hline Transferrina $(\mu \mathrm{g} / \mathrm{dL})$ & $261 \pm$ & $\pm \quad 85,2$ & $255 \pm 81,3$ & $285 \pm 85,1^{d}$ \\
\hline
\end{tabular}

* Número de pacientes = 56; VCM = volume corpuscular médio; HCM hemoglobina corpuscular média; CHCM= concentração de hemoglobina corpuscular média

a Diferença significativa nos valores pré-operatórios vs 6 and 12 mo $(P<0,01)$

${ }^{b}$ Diferença significativa nos valores pré-operatórios vs 6 and 12 mo $(P<0,01)$

c Diferença significativa nos valores pré-operatórios vs 12 mo $(P=0,001)$

${ }^{d}$ Diferença significativa nos valores pré-operatórios vs 6 and 12 mo $(P=0,05)$

e Diferença significativa nos valores pré-operatórios vs 12 mo $(P=0,08)$

Tabela 3 - Percentagem de pacientes com alteração dos parâmetros relacionados à síndrome metabólica antes e após bypass gástrico.

\begin{tabular}{|c|c|c|c|c|c|c|c|}
\hline Parâmetros & $P$ & $\begin{array}{r}\text { Pré } \\
\mathrm{N}\end{array}$ & $\begin{array}{l}\text { operatório } \\
(\%)\end{array}$ & $\begin{array}{c}\text { Pós operatório } 6 \\
\mathrm{~N} \quad(\%)\end{array}$ & 5 meses & $\begin{array}{r}\text { Pós operatório } \\
\mathrm{N}\end{array}$ & $\begin{array}{c}12 \text { meses } \\
(\%)\end{array}$ \\
\hline $\mathrm{IMB}>40 \mathrm{~kg} / \mathrm{m}^{2}$ & & 56 & $(100)$ & $24 \quad(42,9)$ & & 3 & $(5,4)$ \\
\hline Glucose > 110 mg/dL & & 21 & $(37,5)$ & $4 \quad(7,4)$ & & 1 & $(1,8)$ \\
\hline Colesterol HDL $<40$ mg/dL & & 32 & $(57,1)$ & $27 \quad(48,2)$ & & 15 & $(26,8)$ \\
\hline Triacilglicerol >150 mg/dL & & 22 & $(40,7)$ & $10(18,5)$ & & 4 & $(7,4)$ \\
\hline
\end{tabular}

$n=$ número de pacientes. 
triacilglicerol dos pacientes avaliados, após seis e 12 meses após a cirurgia.

\section{DISCUSSÃO}

No presente estudo, a perda de peso verificada nos pacientes foi $34 \%$ do peso total ao final de um ano de acompanhamento ambulatorial, e este valor é semelhante ao encontrado por Valezi et al. ${ }^{11}$, em estudo prévio no mesmo Hospital Universitário, isto é de 37, 5\%. No geral, a literatura refere que perda de peso entre 35 to $40 \%$ ocorre, principalmente, 12 meses após gastroplastia bem sucedida e frequentemente, grande parte deste efeito permanece durante muitos anos $2,4,12$.

Após a GVYR, observa-se redução na ingestão calórica, acompanhada de alterações no padrão dietético habitual dos pacientes, o que explicaria, parcialmente, a significativa perda de peso e IMC, isto é: ingestão reduzida de leite, arroz e principalmente feijão, doces e sorvetes 13,14 , assim como intolerância à carne ${ }^{15,16}$ que ocorreria devido ao déficit de enzimas proteolíticas e ácido clorídrico, necessários para iniciar a digestão no estômago ${ }^{13}$. Por outro lado, os pacientes deste estudo referiam aumento na quantidade de ovos ingeridos.

Dessa forma, os dados obtidos no presente estudo, são comparáveis aos de outros resultados prévios, que salientam que a redução na ingestão alimentar após cirurgia bariátrica não era substituída por uma ingestão de alimentos mais saudáveis, tornando o acompanhamento clínico-nutricional após a cirurgia altamente recomendável para evitar alterações nutricionais e metabólicas ${ }^{17}$ que podem estar relacionados à múltiplas deficiências nutricionais ou reganho de peso corporal.

Com relação aos dados encontrados sobre o metabolismo do ferro, vale ressaltar que embora os valores de ferro sérico se apresentassem dentro dos padrões de normalidade seis e 12 meses após a operação, verificou-se redução significativa na hemoglobina após seis e 12 meses acompanhada também, de redução em 30\% na ferritina sérica e um significativo aumento nos níveis de transferrina após 12 meses, sugerindo uma queda progressiva nos estoques de ferro corporal.

Deficiências de ferro e vitamina B12 são consideradas como as mais frequentes deficiências nutricionais de pacientes submetidos a este tipo de operação ${ }^{18}$. Elas tornam-se mais graves em decorrência das alterações nos hábitos dietéticos que os pacientes apresentam após a gastroplastia, especialmente, em relação à ingestão de carne vermelha. Além disso, redução na secreção de ácido clorídrico e o desvio cirúrgico duodenal, considerado o local mais eficaz de absorção de ferro, contribuiria também para o aparecimento de anemia por deficiência de ferro. A prevalência de anemia por deficiência de ferro, após esse procedimento cirúrgico pode alcançar um percentual superior a $50 \%$ dos pacientes ${ }^{19,20}$, e as mulheres em idade fértil são mais vulneráveis a apresentar esta síndrome ${ }^{16}$, sendo neste caso recomendada suplementação de ferro ${ }^{21}$. No pre- sente estudo, a maioria dos indivíduos era de mulheres na pré-menopausa ( $39 \pm 8,9$ anos), e este fato pode ter contribuído para o progressivo achado de queda nos níveis de hemoglobina e ferritina. As mulheres representavam, aproximadamente, $90 \%$ dos pacientes no presente estudo, e verificou se redução, com significância estatística nos níveis de ferritina tanto após seis meses como também após um ano, estando estes dados de acordo com os da literatura vigente.

A má nutrição protéica pode ocorrer no pós operatório imediato ${ }^{22}$, mas é menos frequente aparecer no longo prazo em pacientes operados ${ }^{15,18}$. Os resultados verificados no presente trabalho são semelhantes ao de estudos prévios, em que não foi mostrado déficit protéico visceral, verificado mediante níveis séricos de albumina e transferrina após um ano de estudo.

Com relação ao perfil lipídico, verificou-se redução no colesterol total e triacilglicerol de aproximadamente $40 \%$ e $72 \%$, respectivamente, após a gastroplastia 7 , sendo que há um conjunto considerável de evidências mostrando que diminuição isolada de colesterol total e/ou triacilglicerol nos pacientes com outras co-morbidades diminui risco de infarto do miocárdio. Brolin et al. ${ }^{23}$ verificaram queda no colesterol total e triacilglicerol e elevação no HDL-colesterol após gastroplastia, e estes achados persistiram por um longo período de tempo naqueles pacientes que mantiveram a perda de peso, mas também nos que apresentaram reganho de peso durante sua evolução. A melhora no perfil de lipídeos verificada mediante diminuição no colesterol total, LDL-colesterol, e triacilglicerol ocorreu também no presente trabalho, e persistiu mesmo após 12 meses do procedimento cirúrgico. Redução no peso corpóreo e nível de triacilglicerol associada com redução na glicemia refletem o impacto positivo da perda de peso sobre a sensibilidade periférica à insulina ${ }^{24}$.

Em relação à atividade inflamatória, a proteína C-reativa é o marcador de inflamação crônica mais utilizado na prática clínica e fator de risco independente de doença cardiovascular ${ }^{25,26}$. Além disso, níveis elevados desta proteína têm sido associados com obesidade e risco de desenvolver diabetes melito tipo $2^{27}$. Dessa forma, perda de peso e redução nos níveis de proteína C-reativa são considerados parâmetros importantes para reduzir o risco de doença cardiovascular em indivíduos obesos ${ }^{26}$. No presente estudo, houve redução na atividade inflamatória verificada mediante queda nos níveis de proteína C-reativa e, resultados semelhantes são referidos em pacientes obesos submetidos a tratamento cirúrgico ${ }^{8}$ ou não cirúrgico ${ }^{28}$ para perda de peso.

Na presente pesquisa, diminuição no nível das proteínas reagentes de fase aguda positiva, proteína Creativa e ferritina e aumento no nível das proteínas reagentes de fase aguda negativa albumina e transferrina permite aos autores reforçarem acerca da importância da perda de peso em reduzir a atividade inflamatória em pacientes com obesidade grave.

Em conclusão, tratamento cirúrgico em pacientes com obesidade grave apresenta efeitos benéfi- 
cos, pois a perda de peso propicia a redução do colesterol total, do LDL-colesterol, do triacilglicerol e da glicemia. Ocorre também melhora na atividade inflamatória, evidenciada principalmente pela diminuição nos níveis de proteína C- reativa e elevação da albuminemia. A melhora verificada no estado metabólico e inflamatório concomitantemente após o tratamen- to cirúrgico, pode reduzir substancialmente as comorbidades associadas com o risco cardiovascular aumentado.

Estudos posteriores são necessários para esclarecer o real papel da transferrina e ferritina como parâmetros do estado do ferro e atividade inflamatória em pacientes submetidos à gastroplastia em Y de-Roux.

\title{
A B S T R A C T
}

\begin{abstract}
Objective: To assess nutritional and metabolic evolution and inflammatory activity in severe obese patients submitted to bariatric surgery. Methods: This prospective study evaluated 56 patients (50 female and 6 male), mean age $40 \pm 9,9$ years, submitted to RYGB. Nutritional, metabolic, and inflammatory parameters were assessed prior to and 12 months postsurgery. Results: It was verified significant decreases in weight loss in relation to baseline values from 138 $\pm 28,8$ to $90 \pm 19,5 \mathrm{~kg}(p<$ $0,0001)$, glucose levels from $116 \pm 47,3$ to $84 \pm 9,8 \mathrm{mg} / \mathrm{dL}(p<0,0001)$, triacylglycerol levels from $137 \pm 61,4$ to $84 \pm 38,6 \mathrm{mg} /$ $d L(p<0,0001)$, and also in total cholesterol from $189 \pm 41,6$ to $166 \pm 36,4 \mathrm{mg} / \mathrm{dL}(p<0,0001)$ and LDL-cholesterol from 119 $\pm 36,1$ to $104 \pm 30,7 \mathrm{mg} / \mathrm{dL}(p<0,0005)$. C-reactive protein levels reduced from 11,33 $\pm 10,82$ to 3,62 $\pm 4,49 \mathrm{mg} / \mathrm{dL}(p<$ $0,0001)$. Although maintenance of iron levels was verified after one year, there was a significant decrease in hemoglobin from $13 \pm 1,3$ to $12 \pm 1,4 \mathrm{~g} / \mathrm{dL}(p<0,01)$, and reduction in ferritin levels, especially in women who showed a decrease from 101,2 $\pm 123,3$ to $85,0 \pm 101,9$ ( $p<0,03)$. Conclusion: Therefore, weigh loss in patients with severe obese after RYGB showed improvement in both metabolic and inflammatory status and may reduce substantially co-morbidities associated with increased cardiovascular risk.
\end{abstract}

Key words: obesity, gastric bypass surgery, weight loss, dyslipidemia, C-reactive protein

\section{REFERENCIAS}

1. Monteiro CA, Conde WL, Popkin BM. Is obesity replacing or adding to undernutrition? Evidence from different social classes in Brazil. Public Health Nutr. 2002; 5(1A):105-12.

2. Mun EC, Blackburn GL, Matthews JB. Current status of medical and surgical therapy for obesity. Gastroenterology. 2001;120(3):669-81.

3. Schneider BE, Mun EC. Surgical management of morbid obesity. Diabetes Care. 2005;28(2):475-80.

4. Pories WJ, Swanson MS, MacDonald KG, Long SB, Morris PG, Brown BM et al. Who would have thought it? An operation proves to be the most effective therapy for adult - onset diabetes mellitus. Ann Surg. 1995;222(3):339-50, discussion 350-2.

5. Christou NV, Sampalis JS, Liberman M, Look D, Auger S, McLean AP et al. Surgery decreases long-term mortality, morbidity, and health care use in morbidly obese patients. Ann Surg. 2004;240(3):416-23; discussion 423-4.

6. Blackburn GL. Solutions in weight control: lessons from gastric surgery. Am J Clin Nutr. 2005; 82(1 Suppl):248S-52S

7. Buchwald $H$, Avidor $Y$, Braunwald $E$, Jensen MD, Pories W, Fahrbach $K$ et al. Bariatric surgery: a systematic review and meta-analysis. JAMA. 2004; 292(14):1724-37. Erratum In: JAMA. 2005; 293(14):1724.

8. Kopp HP, Kopp CW, Festa A, Krzyzanowska K, Kriwanek S, Minar $E$ et al. Impact of weight loss on inflammatory proteins and their association with the insulin resistance syndrome in morbidly obese patients. Arterioscler Thromb Vasc Biol. 2003; 23(6):1042-7. Epub 2003 Apr 24

9. Alvarez-Leite Jl. Nutrient deficiencies secondary to bariatric surgery. Curr Opin Clin Nutr Metab Care. 2004;7(5):569-75

10. World Health Organization: Obesity. Preventing and managing the global epidemic. Report of a WHO consultation on obesity: Geneva; 1998.

11. Valezi AC, Mali J Jr, Brito EM, Marson AC. Gastroplastia vertical com bandagem em Y-de-Roux: análise de resultados. Rev Col Bras Cir. 2004;31(1):49-56.
12. Brolin RE. Bariatric surgery and long-term control of morbid obesity. JAMA. 2002:288(22):2793-6.

13. Brown EK, Settle EA, Van Rij AM. Food intake patterns of gastric bypass patients. J Am Diet Assoc. 1982;80(5):437-43.

14. Kenler HA, Brolin RE, Cody RP. Changes in eating behavior after horizontal gastroplasty and Roux-en-Y gastric bypass. Am J Clin Nutr. 1990;52(1):87-92.

15. Avinoah E, Ovnat A, Charuzi I. Nutritional status seven years after Roux-En-Y gastric bypass surgery. Surgery. 1992;111(2):137-42.

16. Moize V, Geliebter A, Gluck ME, Yahav E, Lorence M, Colarusso T et al. Obese patients have inadequate protein intake related to protein intolerance up to 1 year following Roux-en-Y gastric bypass. Obes Surg. 2003;13(1):23-8.

17. Lindroos AK, Lissner $L$, Sjöström $L$. Weight change in relation to intake of sugar and sweet foods before and after weight reducing gastric surgery. Int J Obesity 1996;20(7):634-43.

18. Fujioka K. Follow-up of nutritional and metabolic problems after bariatric surgery. Diabetes Care. 2005;28(2):481-4.

19. Amaral JF, Thompson WR, Caldwell MD, Martin HF, Randall HT. Prospective metabolic evaluation of 150 consecutive patients who underwent gastric exclusion. Am J Surg. 1984;147(4):468-76.

20. Brolin RE, Gorman JH, Gorman RC, Petschenik AJ, Bradley LJ, Kenler $\mathrm{HA}$ et al. Are vitamin B12 and folate deficiency clinically important after roux-en-Y gastric bypass? J Gastrointest Surg. 1998;2(5):43642.

21. Brolin RE, Gorman JH, Gorman RC et al. Prophylactic iron supplementation after roux-en-Y gastric bypass: a prospective, double-blind, randomized study. Arch Surg. 1998;133(7):740- 4

22. Martínez N, Martinez MJ, Morales MJ Pena E, Fariña MA et al. Perioperative protein malnutrition in bariatric surgery. Clin Nutr. 2001;20(Suppl 3): Abstract:69

23. Brolin RE, Bradley LJ, Wilson AC, Cody RP. Lipid risk profile and weight stability after gastric restrictive operations for morbid obesity. J Gastrointest Surg. 2000;4(5):464-9.

24. Cummings DE, Overduin J, Foster-Schubert KE. Gastric bypass for obesity: mechanisms of weight loss and diabetes resolution. J Clin Endocrinol Metab. 2004;89(6):2608-15 
25. Ridker PM. High-sensitivity C-reactive protein: potential adjunct for global risk assessment in the primary prevention of cardiovascular disease. Circulation. 2001;103(13):1813-8.

26. Ridker PM. Clinical application of C-reactive protein for cardiovascular disease detection and prevention. Circulation. 2003;107(3):363-9.

27. Pradhan AD, Manson JE, Rifai N, Buring JE, Ridker PM. C-reactive protein, interleucin 6 , and risk of developing type 2 diabetes mellitus. JAMA. 2001;286(3):327-34.

28. Tchernof A, Nolan A, Sites CK, Ades PA, Poehlman ET. Weight loss reduces $C$ - reactive protein levels in obese postmenopausal women. Circulation. 2002;105(5):564-9.
Recebido em 02/02/2009

Aceito para publicação em 03/04/2009

Conflito de interesse: nenhum

Fonte de financiamento: nenhuma

\section{Como citar esse artigo:}

Costa LD, Valezi AC, Matsuo T, Dichi I, Dichi JB. Repercussão da perda de peso sobre parâmetros nutricionais e metabólicos de pacientes obesos graves após um ano de gastroplastia em Y-de-Roux. Rev Col Bras Cir. [periódico na Internet] 2010; 37(2). Disponível em URL: http:/ /www.scielo.br/rcbc

\section{Endereço para correspondência:}

Jane Bandeira Dichi

E-mail: dichi@sercomtel.com.br 